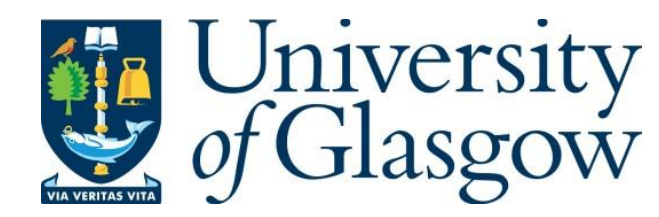

Thring, C.B., Fan, Y. and Edwards, R.S. (2016) Focused Rayleigh wave EMAT for characterisation of surface-breaking defects. NDT \& E International, 81, pp. 20-27.

There may be differences between this version and the published version. You are advised to consult the publisher's version if you wish to cite from it.

http://eprints.gla.ac.uk/175091/

Deposited on: 12 December 2018

Enlighten - Research publications by members of the University of Glasgow http://eprints.gla.ac.uk 


\title{
Focused Rayleigh wave EMAT for characterisation of surface-breaking defects
}

\author{
C.B. Thring, Y. Fan, R.S. Edwards
}

Department of Physics, University of Warwick, Coventry CV4 7AL, United Kingdom

\begin{abstract}
Developments towards higher resolution and the ability to detect small defects are bringing a step-change in non-destructive testing. This paper presents a new method for increasing resolution, using a focused electromagnetic acoustic transducer (EMAT) optimised to generate Rayleigh waves at $2 \mathrm{MHz}$. This high frequency allows detection of $\mathrm{mm}$-depth defects, and the focusing allows sizing of much shorter defects than is possible when using standard EMATs. The focusing behaviour and the aperture angle effect are analysed using laser vibrometry and finite element modeling, showing that a reduced aperture shifts the focal point from the designed value and increases the focal depth. The dual-EMAT has excellent signal to noise ratio (up to $30 \mathrm{~dB}$ ) and has been used in single shot mode to image a variety of surface-breaking defects, including detecting and positioning a pair of real defects in an aluminium billet sample, and a machined defect of $2 \mathrm{~mm}$ length, $0.2 \mathrm{~mm}$ width, and $1.5 \mathrm{~mm}$ depth, giving an upper limit on the defect length of $2.1 \pm 0.5 \mathrm{~mm}$. The results can be used to design an EMAT with optimised focal behaviour for defect detection.
\end{abstract}

Keywords: Ultrasonics, EMAT, Focusing, Rayleigh wave

\section{Introduction}

Non-destructive testing (NDT) and structural health monitoring are essential research areas for industry, with their importance steadily growing as the current infrastructure ages. A 2012 study highlighted required areas of growth, producing a 20-year vision for the field which stressed the importance of increasing sensitivity, with the ability to detect smaller defects and their precursors one of the main requirements of industry [1]. Ultrasonic inspection is one of the fundamental techniques in NDT [2-6]. Non-contact ultrasonic techniques, including laser ultrasonics and electromagnetic acoustic transducers (EMATs), are becoming more widely accepted [4]. These are inefficient when compared to piezoelectric transducers, but this can be mitigated by their lack of requirement

Email address: r.s.edwards@warwick.ac.uk (R.S. Edwards) 
for couplant and the ability to operate in harsh environments out of contact with the sample [3, 7-9].

Surface-breaking defects such as stress corrosion cracking [10] in pipework and rolling contact fatigue (RCF) $[9,11,12]$ on railway tracks can be characterised using surface acoustic waves $[2,3,9,12-15]$. Early stage detection of $\mathrm{RCF}$ requires sensitivity down to around $0.5 \mathrm{~mm}$ depth [16]. Rayleigh waves propagate predominantly within one wavelength of the sample surface [2], hence the sensitivity is dependent on the frequency used for inspection. The lateral resolution is dominated by the lateral extent of the plane waves generated.

Transducers typically used in industry have a large beam profile, limiting the size of defect that can be resolved when using surface-wave techniques. However, if a focused transducer is used, a narrower beam profile and increased signal strength at the focal point is achieved and this can be optimised for defect detection. Rayleigh wave focusing has previously been implemented using shaped piezoelectric elements [18] and with piezoelectric phased arrays [17, 19]. The arrays allow for variable focusing, unlike the single shaped element which has a fixed focal point, but the arrays are costly and complex. Piezoelectric focused transducers have been produced with a beam width of $1.2 \mathrm{~mm}$ [18], while phased arrays have been used to identify holes drilled through an aluminium sample with diameters of 2 and $1 \mathrm{~mm}$ [19].

The use of non-contact ultrasonic methods for generating focused ultrasound beams offers many advantages in terms of implementation of inspection, despite the reduced efficiency of the techniques. The use of optics with laser ultrasound can give a ring shaped beam, which when incident on a sample surface creates an inward traveling wave that becomes focused at the ring center. This focused point has much higher intensity than is possible with a direct incidence laser beam while staying in the thermoelastic (non-destructive) regime of laser generation [20-22]. A ring focused beam has been used to detect a $1 \mathrm{~mm}$ deep, $0.1 \mathrm{~mm}$ width, electro-discharge machined (EDM) slot in aluminium, but this measurement required the defect length to be larger than the ring diameter [21]. Sample surface deposits or screens can also be employed to shape the laser footprint on the sample surface, changing the frequency bandwidth of the generated ultrasound waves and creating a focus [23-25]. However, lasers are not always viable for regular machine testing in a factory setting due to their high costs and potentially serious safety implications.

EMATs are a robust and inexpensive alternative, free of the safety hazards of the laser generation techniques [4]. They can work without direct physical contact to the samples, with no coupling gel required, allowing them to be utilised in a variety of situations where standard piezoelectric ultrasonics are not viable, such as on moving, hot, rough, or rusted surfaces [3, 7-9]. EMATs as generators consist in their simplest form as a coil of wire through which a current is pulsed [4]. This current creates an alternating magnetic field around the coil elements which, when close to a conductive sample, creates a corresponding alternating Lorentz force on the sample's delocalised electrons. These electrons consequently start to oscillate, and the momentum transfer by elastic collision causes their parent ions to move with them. The method can be enhanced 
by the presence of a permanent magnet above the coil. The configuration of the magnetic field direction and the coil shape alters the wavemodes that are generated. EMAT detection works similarly, however it is more efficient than the generation method as it detects the velocity of the electrons themselves rather than the ions [33]. The presence of a permanent magnet is a necessity for detection.

The majority of previous work on focused EMAT techniques have concentrated on shear wave generation [4-6, 26, 27]. A straight meander line EMAT can generate shear waves which propagate into the sample bulk, and varying the meander spacings causes constructive interference of the beam to form a focal line $[4,6]$. Curving this design adds a geometric focus for constructive interference at a point location, and this has been employed for detection of slits deeper than $0.05 \mathrm{~mm}$ on the opposite surface of a $20 \mathrm{~mm}$ thick stainless steel sample [26]. A curved EMAT design for Rayleigh waves has been suggested in EMAT testing standards [28], but this has not been the focus of any concentrated research effort to understand and improve the EMAT behaviour.

This paper details and fully characterises a focused Rayleigh wave dualEMAT design and demonstrates detection of a variety of machined defects and a pair of real defects, showing high resolution surface breaking defect sizing and detection. The effect of the aperture angle on the focus achieved is explained and steps devised which can be used to design an optimised transducer for a chosen focal point.

\section{Method}

The focused concentric dual-EMAT coil, shown in figure 1, was optimised to generate Rayleigh waves at $2 \mathrm{MHz}$ in aluminium, corresponding to a meander line spacing of $1.5 \mathrm{~mm}$. The wire used was $0.08 \mathrm{~mm}$ in diameter and was wound three times through each of the meander line elements. Two separate coils were used, nested together so that they could be used in a pseudo pulse-echo arrangement while allowing for individual impedance matching of the coils. The coils have different aperture angles $\left(11.4^{\circ}\right.$ and $\left.20.1^{\circ}\right)$ as they were designed to fit under the same $35 \mathrm{~mm}$ diameter $\mathrm{NdFeB}$ magnet to facilitate simple scanning and effective alignment with only one transducer. They are individually tuned using capacitors to create an LCR circuit with a resonant frequency of $2 \mathrm{MHz}$ in order to achieve maximum signal strength [4]. This gives a wavelength suitable for detecting defects with depths in the mm range, while still allowing handwinding of the coils to give a high current density. The generation coil has a designed radius of curvature, and hence focal point, of $50.6 \mathrm{~mm}$ when measured from the back edge of the coil.

The dual-EMAT was produced to detect surface-breaking defects with high spatial resolution, with this resolution determined by the beam width at the focal point. In order to reliably detect a defect the beam width should be of the order of, or smaller than, the defect length. By analogy with conventional diffraction-limited optics the theoretical focusing resolution is given by the Abbe 


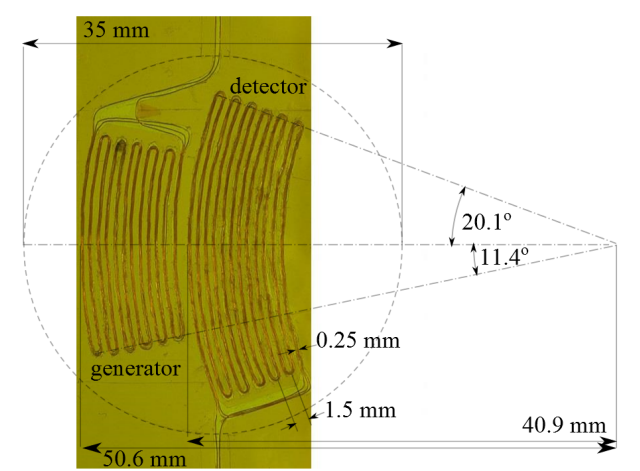

Figure 1: Annotated photograph of the concentric EMAT coils. Dashed circle indicates magnet placement.

diffraction limit[29],

$$
D=\frac{\lambda}{2 \sin \theta}
$$

where $D$ is the minimum width, $\lambda$ is the wavelength of the ultrasound, and $\theta$ is the aperture angle. This predicts $D=3.8 \mathrm{~mm}$ for the generation EMAT.

The generation EMAT coil consists of six meanders. A seven cycle, $2 \mathrm{MHz}$, sinusoidal tone burst was used to generate a surface wave, using an adapted Ritec RAM-5000 pulser-receiver. Signals reflected from defects return as a seven cycle $3.5 \mu$ s long wave packet, and therefore if no signal processing is used the spatial resolution of the technique is limited. However, simple signal processing techniques can be used to extract the wave packet peak to allow accurate position measurements.

To extract the peak of the wave packet in an automated manner, a synthetic signal $G$ with its real component designed to match the generated signal was created:

$$
G=e^{\frac{-\left(t-t_{0}\right)^{2}}{2 a^{2}}} e^{2 i \pi f\left(t-t_{0}\right)}
$$

where $f$ is the frequency, $t_{0}$ is the time offset, and $a$ is the bandwidth of the signal in the time domain. The values were set to match those contained in the real output signal $\left(a=1 \mu \mathrm{s}, f=2 \mathrm{MHz}, t_{0}=20 \mu \mathrm{s}\right)$. This synthetic signal was crosscorrelated with the detected waveforms and the absolute value squared returned, giving the signal power of the wave packet (effectively measuring the peak in the wave envelope), making it possible to find the maximum peak position to a good degree of accuracy. The cross-correlation process also takes advantage of data from all seven cycles of the detected wave as it compares the shape of the full signal packet with $G$, increasing the accuracy of the peak characteristics found, and reducing noise which has different frequency content.

To illustrate the effect this processing has, figure 2 shows an unprocessed, and a processed, A line scan detected by the dual-EMAT when aligned with the focal point at the center of the largest defect used in this study (see the $20 \mathrm{~mm}$ length angled defect outlined in table 1 and figure 3 . The reflection from the 

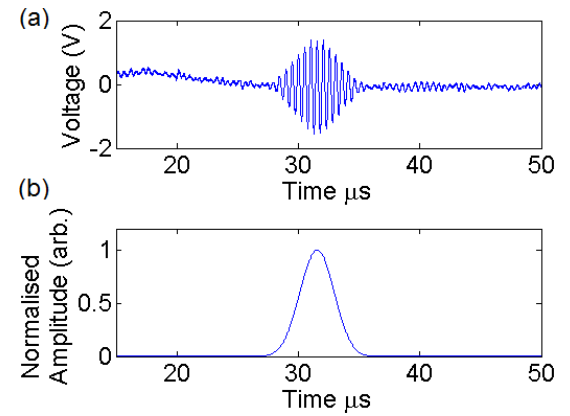

Figure 2: a) Single shot voltage data detected by the EMAT, showing a reflection at the focal point from a large defect. The only processing is amplification and a $0.8-5 \mathrm{MHz}$ bandpass hard filter. b) Shows the same data after cross-correlation with a synthetic signal designed to mimic the generation EMAT output, extracting the signal wave packet.

defect can be seen at just after $30 \mu$ s. The same processing was performed on all the data presented in this work including models, and all of the EMAT detected signals are single shot; no averaging was required due to the high signal to noise ratio (SNR) obtained following EMAT optimisation.

To study the beam profile and the focusing of the generation EMAT, the out-of-plane surface displacement generated on a $21 \mathrm{~mm}$ thick aluminium block was recorded using a laser vibrometer with a spot size of $250 \mu \mathrm{m}$, a spatial resolution of $0.5 \mathrm{~mm}$ and a recording time resolution of $0.1 \mu \mathrm{s}$. As the recording time resolution is low with respect to the $2 \mathrm{MHz}$ wave period $(0.5 \mu \mathrm{s})$, and the spatial resolution is also low with respect to the wavelength $(1.5 \mathrm{~mm})$, hence 128 averages were used to gain a clear image. A numerical simulation was set up using a three dimensional finite element (FE) model implemented in the software package PZFlex. The model was set to have a depth of $10 \mathrm{~mm}$ (approximately $7 \lambda$ ) with the bottom surface boundary condition defined as absorbing in order to best approximate the Rayleigh wave limit of the Rayleigh-Lamb wave dispersion relations [2]. Hexahedron elements of side-length $65 \mu \mathrm{m}$ were used with approximately 23 elements per wavelength, chosen to reduce the artifacts of numerical dispersion caused by higher harmonics in the signal. The same model set up was further employed to find the beam profile dependence on the aperture angle of the coils. The aperture effect was studied experimentally by measuring the amplitude of the generated signals along the focal axis when using either the generator coil $\left(11.4^{\circ}\right.$ aperture), or the detector coil $\left(20.1^{\circ}\right.$ aperture) to generate the signals. A two-wave mixer interferometer was used instead of the laser vibrometer for these final two scans to improve the SNR.

Defect detection requires scanning of the dual-EMAT along / across a sample. The presence of a defect or sample edge is shown by the detection of reflections when it is within the focal length of the transducer. The top $5 \mathrm{~mm}$ of the aluminium sample was machined off prior to testing to remove the layer of material with potential inhomogeneity in the crystallographic texture which is often found in extruded aluminium samples [30]. Five different defects in 


\begin{tabular}{llll}
\hline Defect Type & Depth & Width & Length \\
\hline \multirow{2}{*}{ Angled } & $5 \mathrm{~mm}$ & $\leq 3 \mathrm{~mm}$ & $5 \mathrm{~mm}$ \\
& & & $20 \mathrm{~mm}$ \\
\hline Slot & $1.5 \mathrm{~mm}$ & $\leq 0.2 \mathrm{~mm}$ & $2 \mathrm{~mm}$ \\
\hline Real & $2.7-52 \mathrm{~mm}$ & $\leq 3 \mathrm{~mm}$ & $64 \mathrm{~mm}$ \\
\hline
\end{tabular}

Table 1: Machined defect sizes.

aluminium samples were characterised using the dual-EMAT, with the defect properties summarised in table 1. Cross-sections of the angled and slot defect types are shown in figure 3(b) and (c). These were produced using electrodischarge machining (angled defects) and laser micro-machining (slot defect).

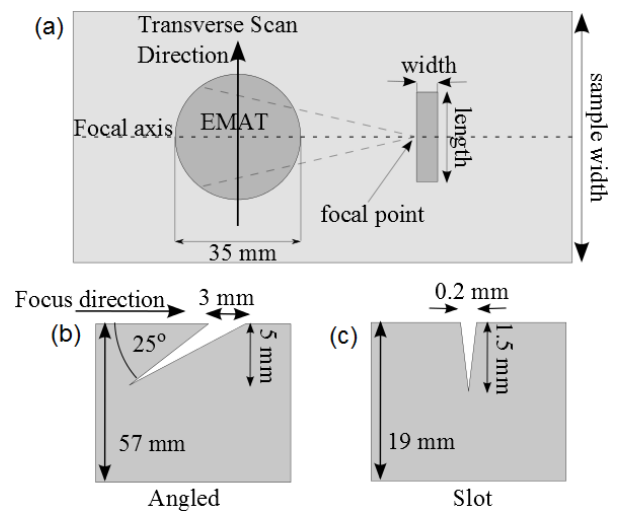

Figure 3: (a) Bird's eye view of sample scanning set up, (b) cross-section of a machined angled defect, (c) cross-section of a laser-micromachined slot defect.

Figure 3(a) shows the schematic for scanning the EMAT either along the focal axis towards a defect or sample edge (dotted horizontal line), or in the transverse direction (arrow). The edge of a large rectangular aluminium sample was used as an infinite depth defect to characterise the focus of the dual-EMAT by finding the point at which the maximum reflected signal (following signal processing) was returned for scans towards the sample edge, along the focusing axis. Defect detection along both scan directions was done, with transverse scans aiming to have the focal axis at the optimum distance from the defect, oriented with the focus towards the defect, as shown in figure 3(a). A real defect in an aluminium billet was also tested. Figure 4 shows a photograph of the sample, which has had part of the surface machined to give an approximately flat surface for testing. The depths of the cracks were measured previously using the alternating current potential drop (ACPD [32]) technique and are shown in 
figure 5 [31]. In all defect scans an automated X-Y stage was used to scan the dual-EMAT with a step size of $0.25 \mathrm{~mm}$, except for the final scan of the real defect which was performed by hand in $1 \mathrm{~mm}$ increments.

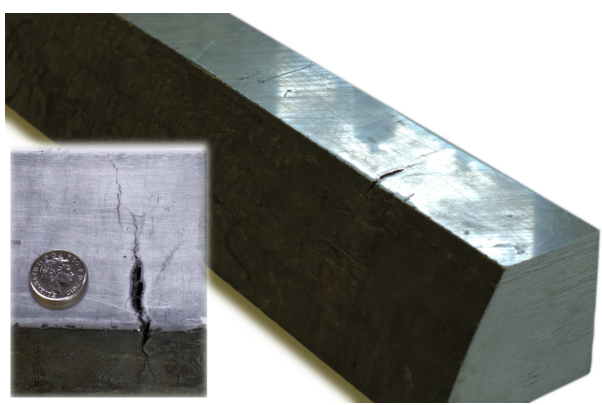

Figure 4: Photographs of an aluminium billet containing two overlapping, real, surface breaking defects. UK 10p coin, $24.5 \mathrm{~mm}$ diameter, shown for size.

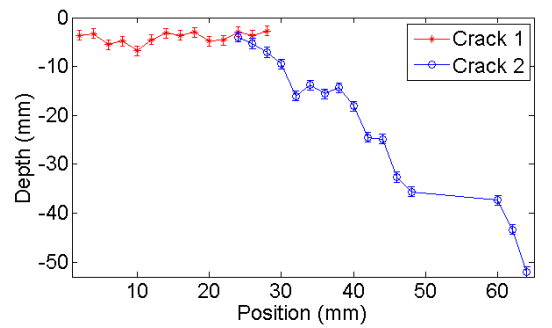

Figure 5: ACPD measured depths of the defects shown in figure 4, data originally from $\mathrm{S}$. Dixon et al [31].

\section{Results and Discussion}

The beam profile generated by the EMAT is shown in figure 6(a). This plot was created by summing the cross-correlated data from each spatial element in the scan over time for $50 \mu \mathrm{s}$, such that regions where the beam passes through appear as bright areas on the figure. The noise speckles in the measured data are due to artifacts of the laser vibrometer setup caused by low reflectivity at certain locations, and the beam is at a slight angle to the horizontal due to a slight error in alignment of the coils with the scan horizontal direction. Note that the cylindrical magnet which forms part of the EMAT can be clearly seen; at these locations the vibrometer is unable to measure the ultrasound propagating within the sample, but instead gives a spurious reading due to the change in height. The out-of-plane displacement of the surface elements given by the FE model was analysed using the same methods as the vibrometer data, and the time summed signal power of the simulated data is shown in figure $6(\mathrm{~b})$. The 


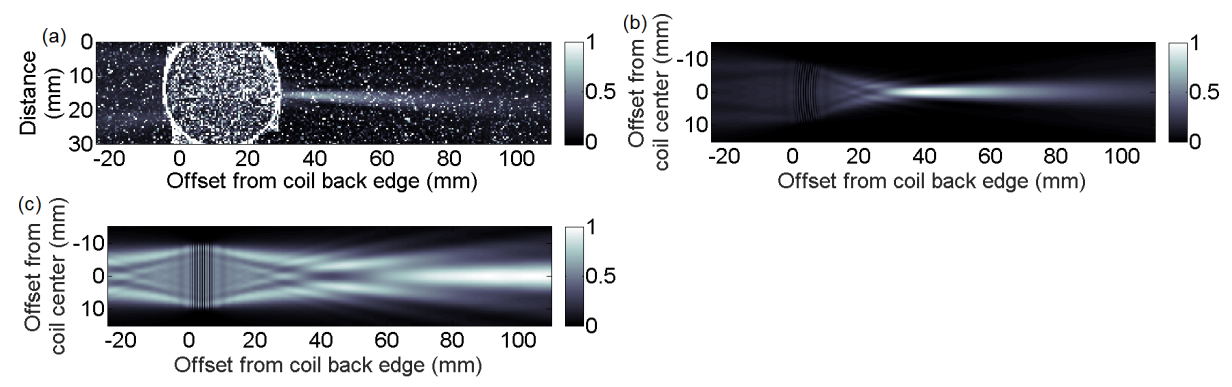

Figure 6: (a) 2D laser vibrometer scan of the surface of an aluminium block $(0.5 \mathrm{~mm}$ spatial resolution). (b) FE model of the scan, (c) FE model of a linear EMAT coil. The grey scale shows normalised signal power (linear scaling from 0 to 1 ) after summation over $50 \mu$ s of time.

model allows measurement of waves underneath and behind the transducer, and the bright regions corresponding to coil positions and a small amplitude backwards travelling wave are just visible on the plot.

The expected focusing effect of the transducer is clear in both experiment and model, with the signal amplitude converging towards the focal point, reaching a maximum and then diverging, clearly distinct to unfocused designs, an example of which has been modelled in figure 6(c). However, the measured focal points were not as expected, found to be at $43 \mathrm{~mm}$ for the vibrometer data, and $40 \mathrm{~mm}$ for the FE model, with an error of $\pm 1 \mathrm{~mm}$ in the experimental data due to the placement and alignment of the EMAT. The unfocused design does have some variation in beam shape due to the near-field effects, but its beam width at a distance of $50 \mathrm{~mm}$ from the generation coil is approximately the same as the linear coil width. The variation in signal strength is also different; the focused coil has a signal strength at the focal point which is much stronger than the initial signal at the generation point, whereas the linear design has a signal strength which remains similar throughout the beam (note that all are normalised to the maximum signal). This shows that the focused design creates a much strong signal at its focal point as well as the tighter beam profile required.

Figure 7 shows the directivity of the signals at distances of radius 43 and $50 \mathrm{~mm}$ from a position at the centre of the back of the generation coil, measured

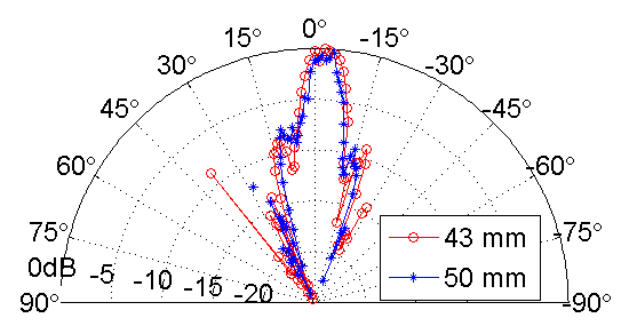

Figure 7: Directivity (polar amplitude distribution) in dB from laser vibrometer scan data. 


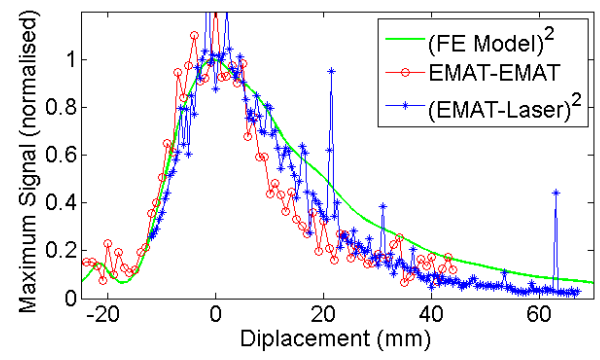

Figure 8: The position of the focus was detected with an EMAT detector (velocity measurement) and a laser vibrometer (displacement measurement) which was also simulated with $\mathrm{FE}$ modelling (displacement modelled). The focal point for each is set as zero displacement.

using the laser vibrometer. The side lobes are about $10 \mathrm{~dB}$ weaker than the main beam and of similar order to the noise. These side lobes are distinguishable in the beam profile for the simulated data (figure 6(b)). The focus is tight at both measurement positions (shown by the angular extent in figure 7 ). The results suggest that the peak signal is occurring slightly earlier than designed (50.6 mm).

The signal amplitude as a function of displacement along the focusing axis is shown in Fig. 8 for the FE model, the vibrometer data, and for scanning the compound EMAT towards the sample edge along the focusing axis. The focal point for each measurement is set to be at zero on the $x$-axis and the approximate fitted peak values are normalised to 1 for comparison. The focused acoustic beam profile from the centerline of the vibrometer detected data (EMAT-laser), and the modeled results (FE Model) do not take into account the focusing of the detector coil and so their values are squared to assume an identical focusing beam profile. It is important to note that the EMAT-EMAT data gives a velocity measurement [33], whereas the FE model and EMAT-laser data give out-of-plane displacements. However, no significant differences were seen in focal position for FE models calculating velocity compared to the displacement models. The expected focusing is clearly observed and the data profiles are in excellent agreement, although the simulated data underestimates the attenuation over longer distances as it neglects the true material attenuation.

The measured focal point of the dual-EMAT was $51 \mathrm{~mm}$ (close to the designed focal length, with an approximate alignment error of $\pm 1 \mathrm{~mm}$ ). The shorter focal length found in the model and the vibrometer data (figure 6) is because the generation coil has a relatively small aperture angle, defined as half of the angular extent of the coil (figure 1), which reduces the focusing effect. Figure 9 shows the focal offset, defined as the difference between the designed and calculated focal lengths, for two designed focal lengths as the aperture angle is varied (FE model); a smaller aperture angle is shown to lead to a shorter apparent focal length. The inset to figure 9 shows the modeled signal power during a scan for several aperture angles (as in figure 8), demonstrating that smaller apertures focus closer to the EMAT but have a longer focal depth, while larger 


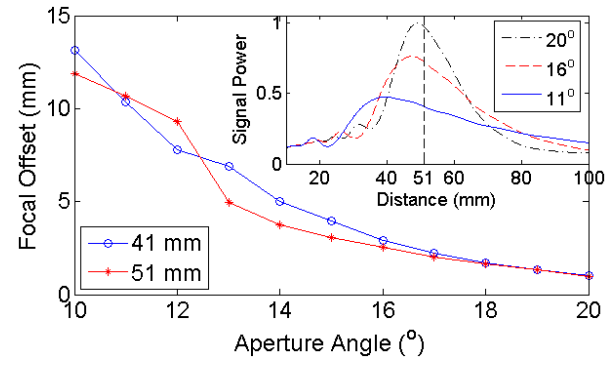

Figure 9: FE models of focal point variation with aperture angle designed for focal lengths of $41 \mathrm{~mm}$ and $51 \mathrm{~mm}$. Inset: signals for coils of varied aperture angles and designed focal length $51 \mathrm{~mm}$.
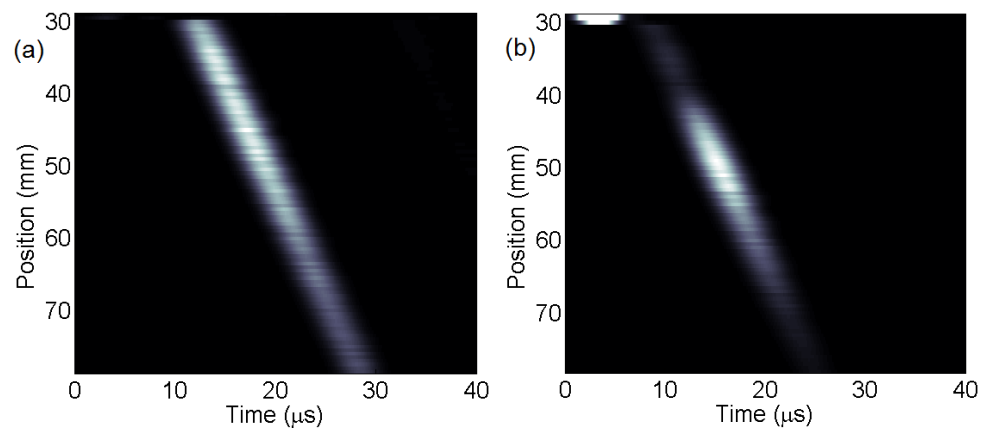

Figure 10: Laser-EMAT B-scan of an aluminium block sample surface for generation using (a) $11^{\circ}$ aperture coil, and (b) $20^{\circ}$ aperture coil. The first three positions show noise only as the laser beam was over the magnet.

apertures focus nearer to the designed focal point with a shorter focal depth.

The aperture effect is confirmed by measurements using each of the EMAT coils in turn to generate a signal. Figure 10 shows B-scans obtained using a twowave mixer interferometer for detection to improve signal to noise ratio. The signal after processing is shown as a function of time and position, with signal amplitude given by the colour scale. The results agree well with the FE model data; the smaller aperture coil has a focal point at $43 \mathrm{~mm}$, in agreement with the laser vibrometer scan, with a large focal depth. The larger aperture coil, used in the dual-EMAT scans as the detector, shows a focal point of $51 \mathrm{~mm}$ with a much narrower focus when used to generate ultrasound, in closer agreement with the coil design. The focusing behaviour of this detection EMAT dominates the achieved focal point in the dual-EMAT measurements due to its shallower focal depth.

An example B scan produced by scanning the dual-EMAT along the focal axis towards the $20 \mathrm{~mm}$ length angled defect is shown in figure 11; the bright regions represent the reflected wave arrival time as the EMAT moves towards the defect. The maximum signal is returned when the back edge of the generation 
coil is $50 \pm 1 \mathrm{~mm}$ away from the start of the defect opening on the surface, in agreement with the measured focal length of the EMAT. The same maximum position was found for all three angled defects. The horizontal position of a defect can be found by looking for the position at which the reflected signal is largest, and for a known ultrasound velocity the time of flight can be used to check the positioning.

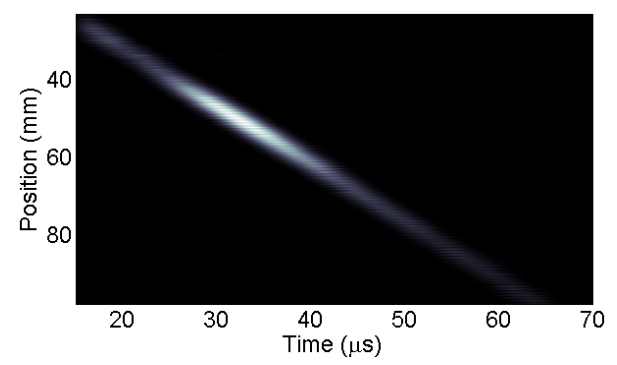

Figure 11: B-scan results from the $20 \mathrm{~mm}$ length defect, characterised in figure 3. Scanning was along the focal axis. Grey scale shows relative signal power.

The transverse scan results from the angled defects are shown in figure 12 as B-scans, for the set-up described in figure 3. The scan was made transversely across the sample at a distance of one focal length from the defects to assess the potential for measuring the defect length. As the defect remains at a fixed position relative to the dual-EMAT the wave arrival time is now constant. SNR was high $(\approx 30 \mathrm{~dB})$ even when working in single shot.

The maximum signal amplitude within the expected arrival time window was found for each EMAT position with results plotted in figure 13. Dashed lines show the actual edges of each defect, and the measurements show a clear correspondence with defect size. The EMAT focal point has a finite width of $3 \mathrm{~mm}$, and hence there is some transitional behaviour around the defect edges where only part of the focal point is incident on the defect and reflected amplitudes are reduced. This causes a rising slope at both sides of all the defects and sizing needs to take account of this. The variation in peak signal strength once the beam is fully incident on the defects is thought to be due to inconsistencies in the inner surface of the defects changing their reflectivity, i.e. showing the effect of surface roughness.

The extent of the rising slopes makes it difficult to pin-point the defect lengths exactly. However, figure 13 shows that it is possible to approximate the defect edge positions by taking the defect extents to be positioned where the signal reaches a set fraction of the maximum reflected signal during the scan. Taking this percentage as $50 \%$ of the maximum signal gives length measurements of $17.9,9.4$, and $4.1 \mathrm{~mm}$ for the 20,10 , and $5 \mathrm{~mm}$ length defects respectively. This underestimates the defect lengths slightly, suggesting that a lower percentage of the peak signal should be used for measuring the lengths. Figure 14 shows how the measured length depends on the percentage used for analysis. Experimentally, a level of 0.3 (or 30\%) returns the most accurate 


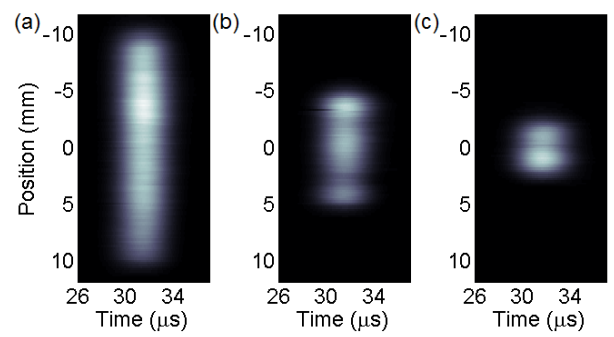

Figure 12: Transverse scans of the angled defects as detailed in figure 3, with lengths of : (a) $20 \mathrm{~mm}$, (b) $10 \mathrm{~mm}$, and (c) $5 \mathrm{~mm}$. Grey scale shows relative signal power.

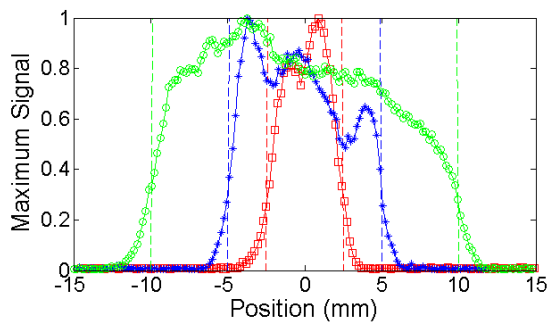

Figure 13: A comparison plot of the maximum returned signal from each position in the transverse scans of the wedge defects outlined in figure 3 , with the dashed lines indicating the actual defect positions.

length measurements for all three defects. The relationship between measured length and percentage is approximately linear between $10 \%$ and $60 \%$ of the maximum signal obtained, and this was used to produce an error by considering measurements within $10 \%$ of the chosen level. This gives length measurements of $20 \pm 0.8 \mathrm{~mm}, 10 \pm 0.4 \mathrm{~mm}$, and $5 \pm 0.5 \mathrm{~mm}$.

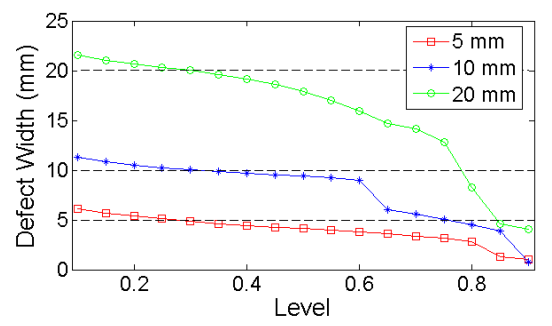

Figure 14: The defect length measurement's dependence on the signal level used to indicate the presence of a defect. Dashed lines indicate actual defect lengths.

The data from the transverse scan of the laser micro-machined slot defect (2 mm long, $1.5 \mathrm{~mm}$ deep) is shown in figure 15. This defect is smaller than the focal size and hence some of the Rayleigh wave is transmitted around and underneath the defect, giving a smaller SNR. The weaker, constant arrival time 


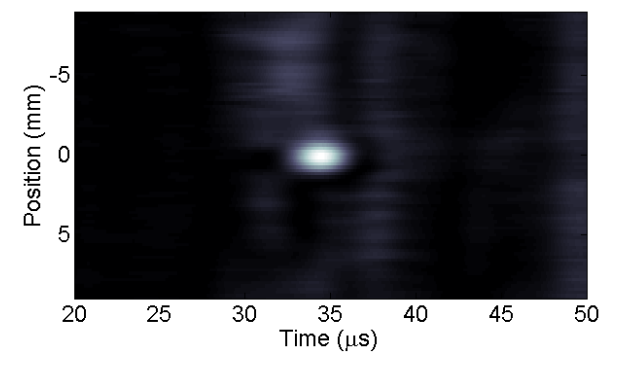

Figure 15: Transverse scan results of the micro-machined slot defect.

(vertical) signals are signals generated within the $\mathrm{NdFeB}$ magnet and its nickel coating, which can be significantly reduced by using improved shielding between coil and magnet. Figure 16 shows the maximum signal found at each position from the scan in figure 15 . The previous data indicates that a level of $30 \%$ of the maximum signal should give the best length measurement, however, as this defect was considerably smaller than the previous ones tested, and is in fact smaller than the beam width of the ultrasound signal, it returns a much weaker signal. In this case a level of $30 \%$ of the peak signal would put the measurement in the background noise. The length of the main signal at a level just above that of the noise, measured at $35 \%$ of the maximum signal, gives a defect length of $2.1 \pm 0.5 \mathrm{~mm}$, giving an upper bound on the size of the defect.

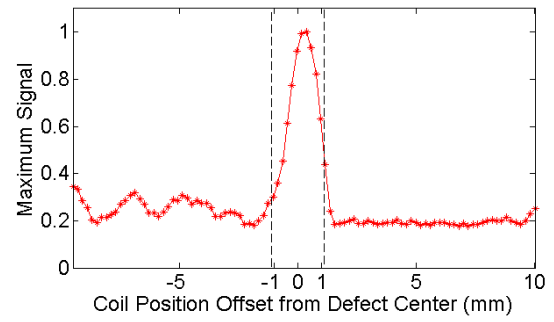

Figure 16: The maximum returned signal at each spatial position from the data in figure 15.

The real defect in an aluminium billet (figures 4 and 5) was also scanned, with figure 17 showing results from a transverse scan. The smaller defect section was set to be approximately at the focal distance from the dual-EMAT. The geometry of the defect on the surface can be seen by the varied times of arrival. The real defect has a much rougher inner surface than the simulated defects used throughout this work, hence the low signal strengths at certain positions.

\section{Conclusions}

The new focused Rayleigh wave dual-EMAT has been fully characterised. The strongest side lobes of the generated Rayleigh wave are approximately $10 \mathrm{~dB}$ 


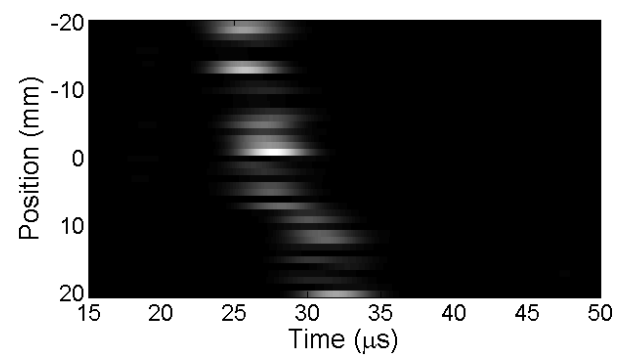

Figure 17: Transverse scan results of the real defect (figure 4).

below the main beam near the focal point, giving a well-focused illumination signal with a minimum beam width at the focal point of $3.0 \pm 0.5 \mathrm{~mm}$. A narrower coil aperture was found to increase the focal depth but give a shorter focal point distance. Optimisation techniques have been used in the design and construction to enable single shot operation. The dual-EMAT has been used for fast scanning of aluminium samples, with a typical SNR of $30 \mathrm{~dB}$. A length measurement accuracy of $0.8 \mathrm{~mm}$ was found on machined $25^{\circ}$ angled defects, with a cut-off signal level of $30 \%$ of the maximum signal during the B-scan giving the best length accuracy. A laser-micro-machined slot defect of length $2 \mathrm{~mm}$, width $0.2 \mathrm{~mm}$, and depth $1.5 \mathrm{~mm}$ was accurately detected to show the resolution potential.

While having a defect at the focal point returns the strongest signal, the relatively long focal depth $(\approx 21 \mathrm{~mm})$ of the generation coil means defects not precisely at the focal point will still return appreciable signals. Defects can then be pin-pointed by using time of flight measurements or a more detailed $2 \mathrm{D}$ raster scan of the sample. As such, reduction of the focal depth is unlikely to greatly enhance defect detection in typical industrial applications. It is, however, possible to optimise the EMAT coils for specific focusing characteristics depending on the application requirements and the focal properties required. Improvements to the EMAT design can be made using knowledge of the aperture effect, ensuring that the smaller generation coil has a focal point equal to that of the detection coil, while keeping both coils small enough to sit under the same magnet without significantly increasing transducer size.

\section{Acknowledgements}

CBT would like to thank EPSRC and the University of Warwick for funding her PhD studies.

[1] P. Thayer, RCNDE industrial members vision for the future requirements for NDE, Insight 54 pp124-127 (2012)

[2] J. L. Rose, Ultrasonic Waves in Solid Media, Cambridge University Press (1999) 
[3] R. J. Dewhurst, C. Edwards, S. B. Palmer, Noncontact detection of surfacebreaking cracks using a laser acoustic source and an electromagnetic acoustic receiver, Applied Physics Letters 49 pp374-376 (1986)

[4] M. Hirao and H. Ogi, EMATs for Science and Industry: Noncontacting Ultrasonic Measurements, Kluwer Academic Publishers, Boston(2003)

[5] G. A. Alers and D. T. Maclauchlan, High frequency, angle beam EMATs for weld inspection, Review of Progress in Quantitative Nondestructive Evaluation 2A pp271-281 (1983)

[6] H. Ogi, M. Hirao and T. Ohtani, Line-focusing electromagnetic acoustic transducers for the detection of slit defects, IEEE Transactions on Ultrasonics, Ferroelectrics, and Frequency Control 46 pp341-346 (1999)

[7] X. Jian, I. Baillie, and S. Dixon, Steel billet inspection using laser-EMAT system, Journal of Physics D: Applied Physics 40 pp1501-1506(2007)

[8] I. Baillie, P. Griffith, X. Jian and S. Dixon, Implementing an ultrasonic inspection system to find surface and internal defects in hot, moving steel using EMATs, 45th Annual British Conference on NDT (2006)

[9] P. A. Petcher, M. D. G. Potter and S. Dixon, A new electromagnetic acoustic transducer (EMAT) design for operation on rail, Non-Destructive Testing and Evaluation International 65 pp1-7 (2014)

[10] K. R. Tretheway, Some observations on the current status in the understanding of stress-corrosion cracking of stainless steels, Materials and Design 29 pp501-507 (2008)

[11] S. L. Grassie, Rolling contact fatigue on the british railway system: treatment, Wear 258 pp1310-1318 (2005)

[12] Y. Fan, S. Dixon, R. S. Edwards and X. Jian, Ultrasonic surface wave propagation and interaction with surface defects on rail track head, NDT \& E International 40 pp471-477 (2007)

[13] M. H. Rosli, R. S. Edwards and Y. Fan, In-plane and out-of plane measurements of rayleigh waves using EMATs for charactersinging surface cracks, NDT \& E International 49 pp1-9 (2012)

[14] S. Boonsang and R. J. Dewhurst, Enhancement of laserultrasound/electromagnetic acoustic transducer signals from Rayleigh wave interation and surface features, Applied Physics Letters 82, pp3348-3350 (2003)

[15] R. S. Edwards, B. Dutton and A. R. Clough, Interaction of laser generated ultrasonic waves with wedge-shaped samples, Applied Physics Letters 100 184102 (2012) 
[16] J. W. Ringsberg, Life prediction of rolling contact fatigue crack initiation, International Journal of Fatigue 23 pp575-586 (2001)

[17] W. A. K. Deutsch, A. Cheng and J. D. Achenbach, Focusing of Rayleigh waves: simulation and experiments, IEEE Transactions on Ultrasonics, Ferroelectrics, and Frequency Control 2A pp333-340 (1983)

[18] B. G. Kim, J. O. Lee and S. Lee, New design and analysis for point-focusing of surface waves in contact testing, IEEE Transactions on Ultrasonics, Ferroelectrics, and Frequency Control 40 pp162-166 (1993)

[19] W. A. K. Deutsch, A. Cheng and J. D. Achenbach, Self-focusing of Rayleigh waves and Lamb waves with a linear phased array, Research in Nondestructive Evaluation 9 pp81-95 (1997)

[20] S. Dixon, T. Harrison, Y. Fan and P. A. Petcher, Thermoelastic laser generated ultrasound using a ring source, Journal of Phyics D: Applied Physics 45175103 (2012)

[21] P. Cielo, F. Nadeau and M. Lamontagne, Laser generation of convergent acoustic waves for materials inspection, Ultrasonics 23 pp55-62 (1985)

[22] X. Wang, M. G. Littman, J. B. McManus, M. Tadi, Y. S. Kim, A. Askar and H. Rabitz, Focused Bulk Ultrasonic Waves Generated by Ring-shaped Laser Illumination and Application to Flaw Detection, Journal of Applied Physics 80 (8) 4274 (1996)

[23] T. Stratoudaki, J. A. Hernandez, M. Clark, and M. G. Somekh, Cheap optical transducers (CHOTs) for narrowband ultrasonic applications, Measurement Science \& Technology 18 pp843-851 (2007)

[24] R. Gutfeld, D. R. Vigliotti, C. S. Ih and W. R. Scott, Thermoelastic hologram for focused ultrasound, Applied Physics Letters 421018 (1983)

[25] H. Kim, K. Jhang, M. Shin and J. Kim, A noncontact NDE method using a laser generated focused Lamb wave with enhanced defect-detection ability and spatial resolution, NDT \& E International 39 pp312-319 (2006)

[26] T. Takishita, K. Ashida, N. Nakamura, H. Ogi and M. Hirao, Develpment of shear-vertical-wave point-focusing electromagnetic acoustic transducer, Japanese Journal of Applied Physics 54, $07 \mathrm{HC} 04$ (2015)

[27] S. Wang, R. Su, X. Chen, L. Kang and G. Zhai, Numerical and experimental analysis of unidirectional meander-line coil electromagnetic acoustic transducers, IEEE Transactions on Ultrasonics, Ferroelectrics, and Frequency Control 60 pp2657-2664 (2013)

[28] American Society for Testing and Materials, Standard practice for ultrasonic examinations using electromagnetic acoustic transducer (EMAT) techniques, (1996) Designation: E 1816 - 96 
[29] A. Yacoubian, Optics Essentials: An Interdisciplinary Guide, CRC Press (2014)

[30] S. Dixon, C. Edwards and S. B. Palmer, Rayleigh wave dispersion on extruded aluminium, Insight 40 pp635-634 (1998)

[31] S. Dixon, B. Cann, D. L. Carroll, Y. Fan and R. S. Edwards, Non-linear enhancement of laser generated ultrasonic Rayleigh waves by cracks, NonDestructive Testing and Evaluation 23 pp25-34 (2008)

[32] W. H. Cullen, Automated Test Methods for Fracture and Fatigue Crack Growth: A Symposium Sponsored by ASTM Committees E-9 on Fatigue and E-24 on Fracture Testing, Pittsburg, PA, Astm International (1983)

[33] X. Jian, S. Dixon and S. B. Palmer, In-plane and out-of-plane particle velocity measurement using electromagetic acoustical transducers, IEEE Ultrasonics Symposium 1-4 pp1276-1279 (2005) 